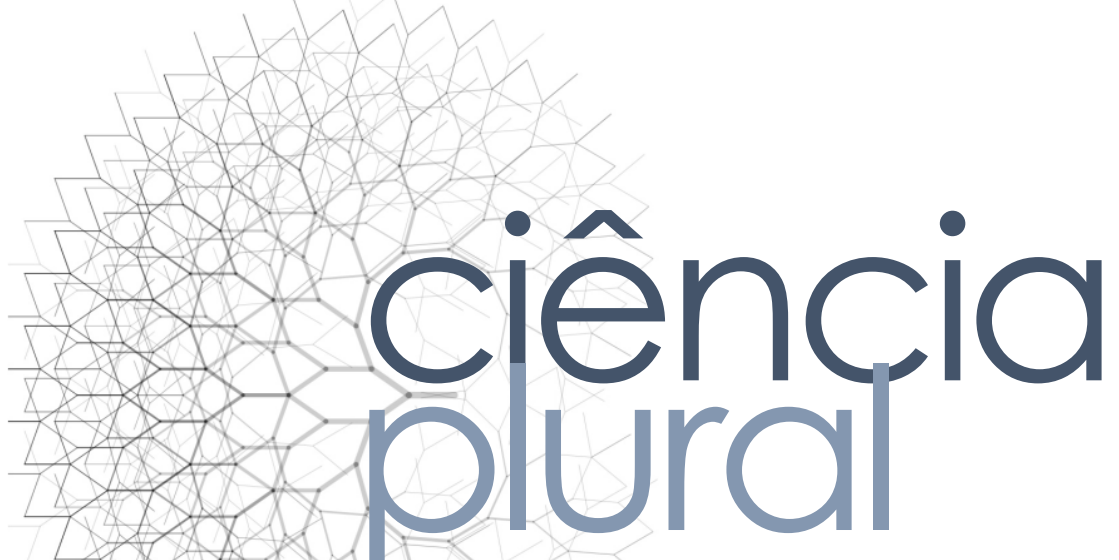

\title{
QUALIDADE DE VIDA, SINTOMAS DE DEPRESSÃO E ADESÃO AO TRATAMENTO EM PACIENTES COM TRANTORNO DEPRESSIVO MAIOR
}

Quality of life, depression symptoms and adherence to treatment in patients with major depressive disorder

Calidad de vida, síntomas depresión y adhesión al tratamiento en pacientes con trastorno depresivo mayor

Pedro Felipe Marques Andrade - Universidade Federal do Rio Grande do NorteUFRN • Departamento de Ciências Farmacêuticas-DCF- PPgCF/UFRN • E-mail: pedrofelipe1125@gmail.com

Karla Simone da Costa de Souza • UFRN • PPgCF/UFRN •

E-mail: karlacostasouza@gmail.com

Georgia Oliveira do Amaral•UFRN • DCF • E-mail: georgiaamaral@hotmail.com

Emerson Arcoverde Nunes • UFRN • Departamento de Medicina Clínica-DMC •

E-mail: emerson_arcoverde@yahoo.com.br

Andréa Cristiane Azevedo Ramos• UFRN • DMC •

E-mail: deia.medicina@gmail.com

Gabriel Queiroz Moura • UFRN• Departamento de Medicina-DM •

E-mail: gabrielqm1@gmail.com

Jayane Cristine Rodrigues de Brito •UFRN•DM・E-mail: jayane.ufrn@gmail.com Wilton dos Santos Rocha • UFRN • DM • E-mail: wilton.sm2@gmail.com Maria Luiza Diniz de Sousa Lopes • Programa de Pós-graduação em Saúde Coletiva-PPGSCol • E-mail: marialu.diniz@gmail.com Aurigena Antunes de Araújo • Programa de Pós-graduação em Ciências Odontológicas • PPgCF/UFRN・E-mail: aurigena@ufrnet.br

Autora correspondente:

Aurigena Antunes de Araujo • E-mail: aurigena@ufrnet.br 


\section{RESUMO}

Introdução: Pacientes com depressão maior geralmente respondem ao tratamento com medicamentos antidepressivos, no entanto em $10 \%$ a $30 \%$ dos casos há apenas uma resposta parcial ou nenhuma resposta, entre os fatores que podem influenciar encontrase o perfil das enzimas hepáticas metabolizadoras dos antidepressivos, tal como a CYP2C19. Objetivo: Caracterizar os indivíduos quanto ao perfil genético dos polimorfismos CYP2C19*2 ou CYP2C19*17 em pacientes com transtorno depressivo maior (TDM) tratados com citalopram ou escitalopram e compará-los em relação a adesão ao tratamento, sintomas de depressão e qualidade de vida. Metodologia: Tratase de um estudo transversal realizado com 29 pacientes com TDM. Amostras de sangue foram coletadas para genotipagem de CYP2C19 por discriminação alélica TaqMan®. Após caracterização do perfil genético, os indivíduos foram comparados quanto aos dados demográfico e socioeconômico, adesão ao tratamento (Teste de Morisky-Green), sintomas de depressão (escala de Hamilton) e qualidade de vida (WHOQoL-BREF). Resultados: Quatro pacientes (13.8\%) apresentaram polimorfismo para CYP2C19*2 e 10 pacientes $(34.4 \%)$ para CYP2C19*17, com maior prevalência de CYP2C19*17 ( $\mathrm{p}>0.05)$. Nenhuma associação significativa de características socioeconômicas, demográficas e clínicas entre os genótipos do CYP2C19. No Teste de Morisky-Green, a adesão moderada ao tratamento foi predominante nos pacientes CYP2C19*2 e CYP2C19*17 (p>0.05). Não foi observada associação entre sintomas de depressão e polimorfismos genéticos ( $p>0.05)$. Uma associação significativa entre o genótipo polimórfico CC do CYP2C19*17 com a satisfação com a saúde, enquanto o genótipo CT foi associado ao estado "nem satisfeito/nem insatisfeito" $(\mathrm{p}<0.05)$. A maioria dos indivíduos CYP2C19*2 e CYP2C19*17 relatou "necessidade de melhorar" em relação aos domínios de qualidade de vida físico, psicológico, social e ambiental $(p>0.05)$. Conclusões: Os pacientes apresentaram maior prevalência do polimorfismo CYP2C19*17, com moderada adesão ao tratamento. Alguns pacientes, mesmo sob efeito da medicação, apresentaram sintomas de depressão moderado a intenso e relataram uma indefinição na satisfação da sua qualidade de vida.

Palavras-Chave: Transtorno Depressivo Maior. Citalopram. Escitalopram. Citocromo P450. CYP2C19.

\section{ABSTRACT}

Introduction: Patients with major depression usually respond to treatment with antidepressant drugs, however in $10 \%$ to $30 \%$ of cases there is only a partial response or no response, among the factors that can influence is the profile of liver enzymes metabolizing antidepressants, such as CYP2C19. Objective: To characterize the individuals regarding the genetic profile of CYP2C19*2 or CYP2C19*17 polymorphisms in patients with major depressive disorder (MDD) treated with citalopram or escitalopram, and to compare them according to treatment adherence, symptoms of depression and quality of life. Methodology: This is cross-sectional study carried out with 29 patients with MDD. Blood samples were collected for CYP2C19 genotyping by TaqMan ${ }^{\circledR}$ allelic discrimination. After characterization of the genetic profile, the duals were compared regarding the demographic and socioeconomic data, nent adherence (Morisky-Green Test), symptoms of depression (Hamilton scale) quality of life (WHOQoL-BREF). Results: Four patients showed (13.8\%) CYP219*2 10 patients $(34.4 \%)$ CYP219*17 polymorphisms.,with higher prevalence of 


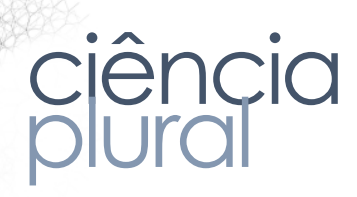

CYP219*17 ( $\mathrm{p}>0.05)$. No association between socioeconomic, demographic, and clinical features with CYP2C19 genotypes was observed. In Morisky-Green Test, moderate adherence to treatment was predominant for CYP2C19*2 and CYP219*17 patients $(p>0.05)$. No statistically significant association was observed between symptoms of depression and genetic polymorphisms $(p>0.05)$. A significant association between polymorphic CC genotype of CYP219* 17 with health satisfaction, while the CT genotype was associated with "neither satisfied/nor dissatisfied" status $(\mathrm{p}<0.05)$. Most of the CYP2C19*2 and CYP2C19*17 subjects reported "need to improve" or "regular" regarding physical, psychological, social, and environmental domains of quality of life ( $\mathrm{p}>0.05)$. Conclusions: The patients showed a higher prevalence of CYP219*17 polymorphism, with moderate treatment adherence. Some subjects, even under the effect of the medication, presented moderate to intense symptoms of depression, and reported a lack of definition in the satisfaction of their quality of life .

Keywords: Major depressive disorder. Citalopram. Escitalopram. Cytochrome P-450. CYP2C19.

\section{RESUMEN}

Introducción: Los pacientes con depresión mayor responder al tratamiento con antidepresivos, en 10\% al 30\% de los casos existe una respuesta parcial o nula, entre los factores que pueden influir se encuentra el perfil de enzimas hepáticas metabolizadoras de antidepresivos, como CYP2C19. Objetivo: Caracterizar a los individuos en cuanto al perfil genético de polimorfismos CYP2C19 * 2 o CYP2C19 * 17 en pacientes con trastorno depresivo mayor (TDM) tratados con citalopram o escitalopram y compararlos en relación para la adherencia al tratamiento, síntomas de depresión y la calidad de vida. Metodología: Estudio transversal con 29 pacientes con TDM. Se recogieron muestras de sangre para la determinación del genotipo CYP2C19 mediante discriminación alélica TaqMan ${ }^{\circledR}$, los individuos fueron comparados en cuanto a los datos demográficos y socioeconómicos, adherencia (Prueba de Morisky-Green), síntomas de depresión (escala de Hamilton) y calidad de vida (WHOQoL-BREF). Resultados: Cuatro pacientes $(13,8 \%)$ con polimorfismo CYP2C19*2 y $10(34,4 \%)$ con CYP2C19* 17,(p> 0,05). No existe una asociación significativa de las características socioeconómicas, demográficas y clínicas con los genotipos CYP2C19. La adherencia moderada al tratamiento fue predominante en los pacientes con CYP2C19*2 y CYP2C19*17 ( $p>0,05)$. No hubo asociación entre síntomas de depresión y polimorfismos genéticos $(\mathrm{p}>0.05)$. Una asociación significativa entre el genotipo polimórfico CYP2C19 * 17 CC con la satisfacción con la salud, mientras que el genotipo CT se asoció con el estado "ni satisfecho / no insatisfecho" (p <0.05). La mayoría de CYP2C19 * 2 y CYP2C19 * 17 individuos informaron "necesidad de mejorar" en relación con los dominios físico, psicológico, social y ambiental de calidad de vida ( $\mathrm{p}>$

0,05). Conclusiones: Los pacients mostraron una mayor prevalencia del CYP2C19 * 17, con adherencia moderada al tratamiento, síntomas de depresión moderada a intensa y

informaron una falta de definición en la satisfacción de su calidad de vida

pras clave: Trastorno depresivo mayor. Citalopram. Escitalopram. Citocromo P450. CYP2C19. 


\section{Introdução}

O transtorno depressivo maior (TDM) é um transtorno mental comum que pode resultar em deficiências graves que afetam o funcionamento psicossocial e a qualidade de vida. Em 2008, a OMS classificou o TDM como a terceira causa de carga de doença em todo o mundo e estimou que ocupará o primeiro lugar até 20301. No Brasil, a taxa de prevalência de depressão é de $4 \%$, afetando principalmente mulheres, pessoas com idades entre 40-59 anos ou 80 anos ou mais, residentes em áreas urbanas, com menor escolaridade, tabagistas e portadores de doenças crônicas como hipertensão arterial, diabetes e cardiopatias ${ }^{2}$.

Existem várias modalidades terapêuticas para o TDM, sendo os antidepressivos a abordagem mais utilizada. Os antidepressivos têm eficácia semelhante, com diferenças importantes em relação à tolerabilidade e segurança ${ }^{3}$. Pacientes com depressão maior geralmente respondem ao tratamento antidepressivo, mas em $10 \%$ a $30 \%$ dos casos há apenas uma resposta parcial ou nenhuma resposta junto com comprometimento funcional, diminuição da qualidade de vida, comportamento autolesivo, pensamentos e tentativas de suicídio e um alta taxa de recaída ${ }^{4}$.

O citalopram e o escitalopram fazem parte da classe de antidepressivos inibidores seletivos da recaptação da serotonina (ISRS), frequentemente usados como tratamento de primeira linha para a depressão devido à sua eficácia, tolerância e segurança 5 . As enzimas hepáticas do citocromo P450 (CYP450) participam do metabolismo de diversos fármacos, sendo as isoenzimas CYP2C19 responsáveis por grande parte do metabolismo do citalopram e do escitalopram ${ }^{6}$.

As atividades da enzima CYP2C19 são influenciadas por muitos polimorfismos em seus genes codificadores de proteínas. Aproximadamente 2.000 polimorfismos de nucleotídeo de referência única (rsSNPs) foram identificados no gene CYP2C19 e mais de 28 alelos foram registrados no Comitê de Nomenclatura de Alelos ${ }^{7}$. O alelo CYP2C19 *1, tipo selvagem, codifica uma enzima funcional que caracteriza o extenso enótipo de metabolização. Um dos principais polimorfismos é a variante CYP2C19 * associada a uma atividade ultrarrápida da enzima devido a uma transição de sina (C) para timina (T) na região 50 flanqueadora do gene CYP2C19. Por outro , o alelo CYP2C19 * 2 resulta de uma transição de guanina $(G)$ para adenina $(A)$ na 
posição 681 no exon 5 e está associado à perda da atividade enzimática de CYP2C198. Indivíduos com variantes do CYP2C19*2 e CYP2C19*17 são mais suscetíveis ao risco de falha terapêutica, com menor probabilidade de remissão dos sintomas depressivos ou não adesão à terapia 9 .

O conhecimento abrangente do polimorfismo contribuirá para o ajuste da dose, o que resulta em eficácia clínica, com controle dos sintomas e adesão ao tratamento, e consequentemente contribuirá para a qualidade de vida do paciente com depressão. O presente estudo teve como objetivo caracterizar os indivíduos quanto ao perfil genético dos polimorfismos CYP2C19*2 ou CYP2C19*17 em pacientes com transtorno depressivo maior (TDM) tratados com citalopram ou escitalopram e compará-los em relação a adesão ao tratamento, sintomas de depressão e qualidade de vida.

\section{Metodologia}

\section{Desenho do estudo e aspectos éticos}

Trata-se de um estudo transversal, realizado com pacientes ambulatoriais da psiquiatria e psicodermatologia do Hospital Universitário Onofre Lopes (HUOL) da cidade de Natal, RN, Brasil. Este estudo foi aprovado pelo Comitê de Ética em Pesquisa da UFRN (Protocolo $\left.n^{0} 71427717.0 .000 .5292\right)$. A participação foi voluntária e todos os participantes assinaram o Termo de Consentimento Livre e Esclarecido. Todos os procedimentos realizados estavam de acordo com os padrões éticos do comitê de pesquisa institucional e nacional e com a declaração de Helsinque.

\section{Amostragem}

Para calcular o tamanho de amostra apropriado, usamos um intervalo de confiança de $95 \%$ e um erro de amostragem tolerável de 15\%. Considerando a população infinita e a prevalência de $42,9 \%$ de depressão em idosos 11 , foi utilizada a seguinte fórmula:

$$
\mathrm{n}=\mathrm{z}_{\mathrm{\alpha} / 2} \mathrm{P}(1=\mathrm{P}) / \varepsilon^{2},
$$

nde $\mathrm{n}$ é o tamanho da amostra, za / 2 é o intervalo de confiança, P é a prevalência e o erro de amostragem tolerável. De acordo com esse cálculo, o tamanho da amostra cessário era de 40 pacientes, que aumentamos em aproximadamente 20\% -DEFF EFF é o efeito do desenho, que é um ajuste que deve ser usado para determinar o 
tamanho da amostra da pesquisa). Objetivamos, portanto, incluir 50 participantes com depressão. A amostragem não probabilística por conveniência foi utilizada para selecionar os indivíduos entre aqueles que aguardavam atendimento.

Os pacientes atendidos nos ambulatórios de psiquiatria e psicodermatologia localizados no HUOL, com diagnóstico de TDM de acordo com os critérios do DSM 5 (Manual Diagnóstico e Estatístico de Transtornos Mentais) e tratados com citalopram ou escitalopram durante o episódio depressivo atual (média de 12 semanas) nos anos de 2019 foram convidados a participar.

Os critérios de exclusão foram pessoas menores de 18 anos; mulheres grávidas ou amamentando; com diagnóstico primário ou comorbidade com esquizofrenia, transtorno esquizoafetivo, transtorno bipolar, demência, doenças neurológicas como encefalopatia, deficiência intelectual ou epilepsia e doenças médicas clinicamente significativas; presença de dependência de álcool ou substâncias ilícitas, com base nos critérios do DSM 5; resultados laboratoriais anormais na triagem; e pessoas que usam drogas que podem exercer influência sobre a atividade do gene CYP2C19. As pontuações do Mini Exame do Estado Mental (MEEM) abaixo dos seguintes limiares de acordo com o nível de escolaridade foram excluídas do estudo: $<11$ pontos para analfabetos, $<22$ pontos para aqueles com 1-11 anos de estudo e $<27$ pontos para aqueles com > 11 anos de educação. Após abordagem e identificação dos indivíduos pelos critérios de elegibilidade, a amostra final constou de 29 indivíduos.

\section{Coleta de dados}

Após a aplicação dos critérios de elegibilidade, bem como a assinatura do termo de consentimento livre e esclarecido, os indivíduos foram submetidos a exames clínicos para confirmação do diagnóstico de TDM, todos devidamente validados e aplicados por psiquiatras.

A Escala de Avaliação de Depressão de Hamilton (HAM-D), criada por Max Hamilton et al. ${ }^{10}$, é o padrão ouro para estudos de validação de outros testes. A versão mais utilizada do HAM-D é composta por 17 itens, sendo hetero-aplicada durante uma trevista clínica para avaliar a remissão dos sintomas depressivos. 
Além disso, pesquisadores previamente treinados e calibrados entrevistaram os participantes e aplicacaram os instrumentos de pesquisa. Usou-se um questionário socioeconômico e demográfico para coletar dados sobre idade, sexo, escolaridade, ocupação, estado civil, tipo de moradia e renda familiar;

Variáveis clínicas também foram investigadas: diagnóstico de depressão, uso de antidepressivo anterior, motivo da troca do antidepressivo anterior, antidepressivo atual, tempo de uso do antidepressivo atual, efeitos adversos, apoio familiar e acesso a consultas.

O Teste de Morisky-Green é o questionário mais utilizado no Brasil para avaliação de adesão ao tratamento, sendo útil na diferenciação entre baixa adesão e não resposta ao tratamento utilizado. É um questionário heteroaplicado, de fácil aplicação e rápido execução, constituído de 04 perguntas, classificando o paciente em aderente (nenhuma questão positiva), moderada adesão (uma a duas positivas) e baixa adesão (três a quatro respostas). O Teste de Morisky-Green ${ }^{11}$ foi utilizado para identificar a adesão ao tratamento, a partir das seguintes perguntas: 1) Você, alguma vez, esquece de tomar seu remédio? 2) Você, às vezes, é descuidado quanto ao horário de tomar seu remédio? 3) Quando você se sente bem, alguma vez, você deixa de tomar o remédio? 4) Quando você se sente mal com o remédio, às vezes, deixa de tomá-lo?

A versão abreviada do questionário de qualidade de vida da Organização Mundial da Saúde (WHOQOL-BREF) foi usada para avaliar a qualidade de vida. Fleck et al. ${ }^{12}$ traduziram o instrumento para o português brasileiro e o validaram em uma população brasileira. Esse instrumento possui 26 itens: duas questões gerais sobre qualidade de vida e satisfação com a saúde e 24 questões nos domínios físico, psicológico, ambiental e relações sociais. Os escores médios dos domínios são calculados, com escores mais altos indicando melhor qualidade de vida.

\section{Genotipagem}

Após responder e preencher as escalas e questionários, os pacientes foram companhados até o laboratório de coleta do HUOL, onde as amostras de sangue ram coletadas por punção a vácuo da veia braquial. O DNA foi obtido de leucócitos sangue periférico usando QiaAmp DNA Blood Mini Kit ${ }^{\circledR}$ (Qiagen, Hilden, manha), de acordo com o protocolo do fabricante. A integridade do DNA foi 
avaliada por separação eletroforética em gel de agarose 0,8\% em tampão Tris-boratoEDTA (TBE) $(\mathrm{pH}=8,0)$ e corado com GelRedTM (Uniscience, São Paulo, SP, Brasil). O DNA foi quantificado (A260nm) e teve sua pureza (A260 / A280) avaliada por espectrofotometria ultravioleta, por meio do espectrofotômetro ND-1000 (NanoDrop Technologies Inc., Wilmin). A genotipagem objetiva as seguintes distribuições de genótipos e frequências de alelos para CYP2C19 * 1B 99 C> T, CYP2C19 * 2681 G> A e CYP2C19 * $17-806$ C > T.

O polimorfismo 99C>T (rs17885098) do alelo CYP2C19*1B, caracterizado pela substituição de uma citocina $(\mathrm{C})$ por uma timina $(\mathrm{T})$ confere o genótipo tipo selvagem para CYP2C19. Portanto, pacientes portadores dos genótipos TC e TT possuem função normal enzimática da CYP2C19, não comprometendo a metabolização dos medicamentos. Entretanto, pacientes com o genótipo CC para a variante CYP2C19*1B apresentam maior risco de alterações enzimáticas da CYP2C19 se associado com os polimorfismos do alelo CYP2C19*2 e CYP2C19*17.

Entre os polimorfismos do CYP2C19 que podem alterar a metabolização de medicamentos encontra-se o 681G>A (rs4244285) do alelo CYP2C19*2, que é caracterizado pela substituição de uma guanina $(G)$ por uma adenina (A) Genótipos AG e AA dessa variante são associados à redução da atividade enzimática da CYP2C19 e, consequentemente ao metabolismo lento dos medicamentos.

Um outro polimorfismo dessa isoenzima que compromete a metabolização de fármacos do tratamento do transtorno depressivo maior é o -806C> T (rs12248560) do alelo CYP2C19*17, o qual é resultante da substituição de uma citosina (C) por uma timina (T). Os genótipos CT e TT para essa variante estão associados a um fenótipo metabolizador ultrarrápido da enzima CYP2C19, que se traduz em eliminação mais rápida do medicamento em pacientes que recebem tratamento com dose padrão, ocasionado a falha terapêutica, e assim, necessitando correções posológicas para alcançar a eficácia terapêutica.

\section{álise de dados}

A distribuição genotípica e frequência alélica do CYP219*1B 99C>T, CYP219*2 681G>A e CYP219*17 -806C>T em pacientes com TDM foram apresentadas em 
distribuição absoluta e percentual. Após conhecido o perfil genotípico, os indivíduos foram distribuídos comparados quanto as características demográfico, sociais, econômicas, clínicas e escala de avaliação de depressão de Hamilton (HAM-D), adesão ao tratamento e avaliação da qualidade de vida, através do teste do Qui-Quadrado com nível de significância de 5\%.

\section{Resultados}

As distribuições de genótipos e frequências de alelos para CYP2C19 * 1B 99C> T, CYP2C19 * 2 681G> A e CYP2C19 * 17 -806 C> T são demonstradas na Tabela 1.

Tabela 1. Distribuição genotípica e frequência alélica do CYP219*1B 99C>T, CYP219*2 681G>A e CYP219*17 -806C>T em pacientes com Transtorno Depressivo Maior. Natal RN, 2021.

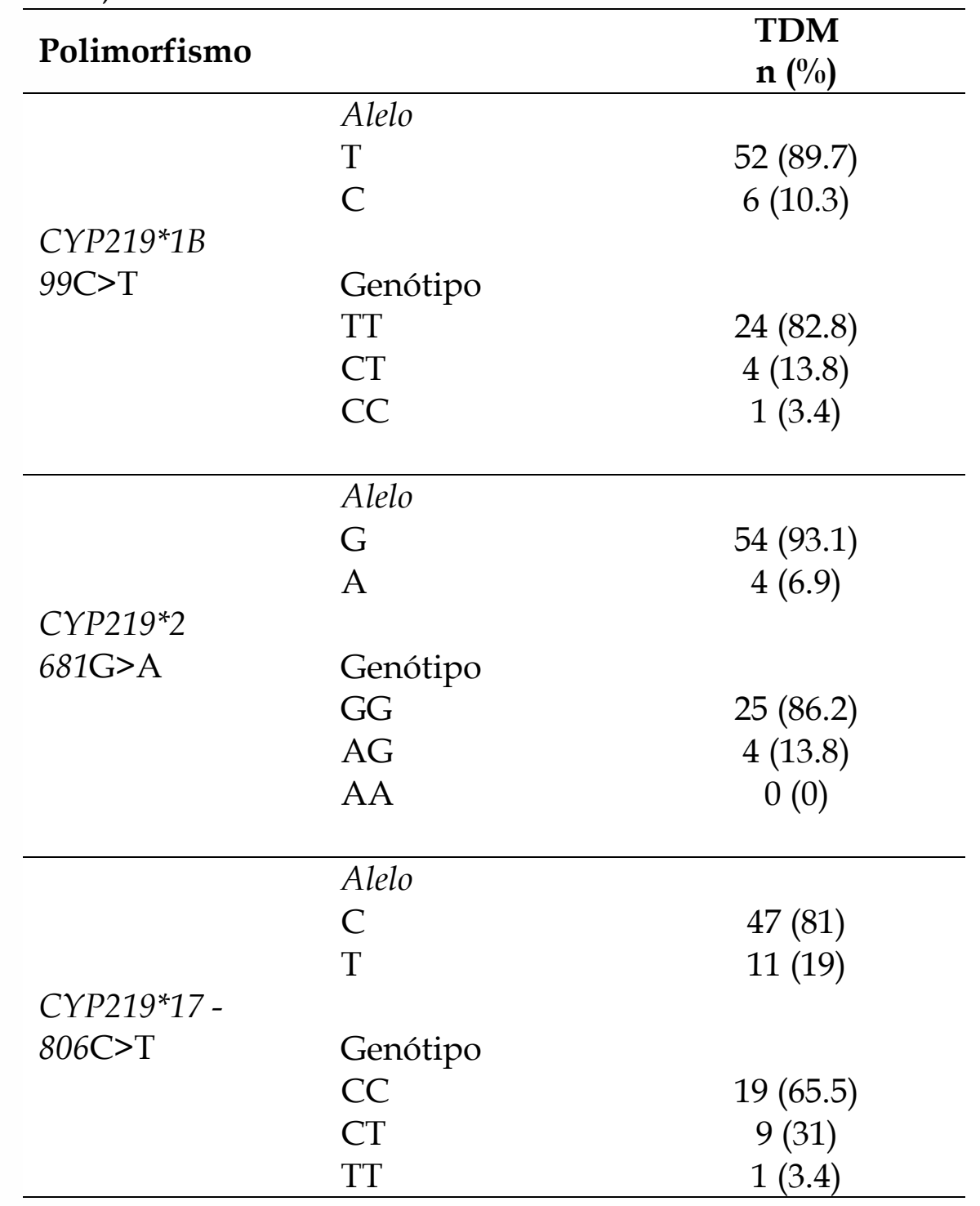


Todos os 29 pacientes responderam aos instrumentos de pesquisa; no entanto, algumas respostas foram indeterminadas porque eles não sabiam a resposta ou não queriam responder. Para os participantes, o polimorfismo no CYP2C19*2 foi confirmado em 4 (13,8\%), enquanto o polimorfismo no CYP2C19*17 foi encontrado em $10(34,4 \%)$ pacientes.

A Tabela 2 mostra a comparação genotípica quando as características demográficas, sociais e econômicas. A maioria dos indivíduos com polimorfismo CYP2C19*2 tinha 18-44 anos, eram de mulheres, desempregadas, com 1-11 anos de estudo e residindo em habitação privada. Os indivíduos com polimorfismo CYP2C19*17 apresentaram perfil semelhante, sendo que a maioria relatou ser solteira e viver com 1 salário-mínimo. Houve uma associação significativa entre os genótipos polimórficos CC e CT do CYP2C19*17 com indivíduos que residiam em habitações privadas $(\mathrm{p}=0,015)$. 


\section{ciência
purál}

Tabela 2. Comparação entre os polimorfismos genéticos de acordo com características demográficas, sociais e econômicas. Natal RN, 2021.

\begin{tabular}{|c|c|c|c|c|c|c|c|c|c|c|c|}
\hline \multirow{2}{*}{ Variáveis } & \multicolumn{4}{|c|}{ CYP219*1B (normal) } & \multicolumn{3}{|c|}{ CYP219*2 (lento) } & \multicolumn{4}{|c|}{ CYP219*17 (ultra-rapido) } \\
\hline & $T T$ & $C T$ & $C C$ & p-valor & GG & $A G$ & p-valor & $C C$ & $C T$ & $T T$ & p-valor \\
\hline Gênero & & & & & & 4 & & & & & \\
\hline Feminino & $18(62.06 \%)$ & $3(10.34 \%)$ & 0 & 0.257 & $17(58.62 \%)$ & $(13.79 \%)$ & 0.184 & $14(48.27 \%)$ & $6(20.68 \%)$ & $1(3.44 \%)$ & 0.761 \\
\hline Masculino & $6(20.68 \%)$ & $1(3.44 \%)$ & $1(3.44 \%)$ & & $8(27.58 \%)$ & 0 & & $5(17.24 \%)$ & $3(10.34 \%)$ & 0 & \\
\hline Idade (anos) & & & & & & 3 & & & & & \\
\hline $18-44$ & $10(34.48 \%)$ & $3(10.34 \%)$ & $1(3.44 \%)$ & 0.268 & $11(37.93 \%)$ & $(10.34 \%)$ & 0.249 & $9(31.03 \%)$ & $5(17.2 \%)$ & 0 & 0.568 \\
\hline $45-60$ & $14(48.27 \%)$ & $1(3.44 \%)$ & 0 & & $14(48.27 \%)$ & $1(3.44 \%)$ & & $10(34.48 \%)$ & $4(13.79 \%)$ & $1(3.44 \%)$ & \\
\hline \multicolumn{12}{|l|}{ Educação } \\
\hline $1-11$ anos & $20(68.96 \%)$ & $4(13.79 \%)$ & $1(3.44 \%)$ & & $21(72.41 \%)$ & $\begin{array}{c}4 \\
(13.79 \%)\end{array}$ & & $16(55.17 \%)$ & $8(27.58 \%)$ & $1(3.44 \%)$ & \\
\hline$>11$ anos & $3(10.34 \%)$ & 0 & 0 & & $3(10.34 \%)$ & 0 & & $2(6.89 \%)$ & $1(3.44 \%)$ & 0 & \\
\hline \multicolumn{12}{|l|}{ Estado civil } \\
\hline Solteiro & $10(34.48 \%)$ & $1(3.44 \%)$ & $1(3.44 \%)$ & 0.648 & $10(34.48 \%)$ & $2(6.89 \%)$ & 0.861 & $6(20.68 \%)$ & $5(17.2 \%)$ & $1(3.44 \%)$ & 0.662 \\
\hline Casado & $11(37.93 \%)$ & $2(6.89 \%)$ & 0 & & $11(37.93 \%)$ & $2(6.89 \%)$ & & $10(34.48 \%)$ & $3(10.34 \%)$ & 0 & \\
\hline Divorciado & $1(3.44 \%)$ & $1(3.44 \%)$ & 0 & & $2(6.89 \%)$ & 0 & & $1(3.44 \%)$ & $1(3.44 \%)$ & 0 & \\
\hline Viuvo & $2(6.89 \%)$ & 0 & 0 & & $2(6.89 \%)$ & 0 & & $2(6.89 \%)$ & 0 & 0 & \\
\hline \multicolumn{12}{|l|}{ Atividade } \\
\hline Econômica & & & & 0.070 & & 3 & & & & & \\
\hline Desempregado & $18(62.06 \%)$ & $1(3.44 \%)$ & 0 & & $16(55.17 \%)$ & $(10.34 \%)$ & 0.821 & $13(44.82 \%)$ & $6(20.68 \%)$ & 0 & 0.091 \\
\hline Empregado & $4(13.79 \%)$ & $3(10.34 \%)$ & $1(3.44 \%)$ & & $7(24.13 \%)$ & $1(3.44 \%)$ & & $6(20.68 \%)$ & $1(3.44 \%)$ & $1(3.44 \%)$ & \\
\hline Aposentado & $2(6.89 \%)$ & 0 & 0 & & $2(6.89 \%)$ & 0 & & 0 & $2(6.89 \%)$ & 0 & \\
\hline Residencia & & & & & & 3 & & & & & \\
\hline Casa própria & $19(65.51 \%)$ & $4(13.79 \%)$ & $1(3.44 \%)$ & 0.533 & $21(72.41 \%)$ & $(10.34 \%)$ & 0.658 & $18(62.06 \%)$ & $6(20.68 \%)$ & 0 & $0.015^{*}$ \\
\hline Casa Alugada & $5(17.2 \%)$ & 0 & 0 & & $4(13.79 \%)$ & $1(3.44 \%)$ & & $1(3.44 \%)$ & $3(10.34 \%)$ & $1(3.44 \%)$ & \\
\hline \multicolumn{12}{|l|}{ Renda Familiar } \\
\hline 1 salário mínimo & $9(31.03 \%)$ & 0 & 0 & 0.521 & $7(24.13 \%)$ & $2(6.89 \%)$ & 0.512 & $3(10.34 \%)$ & $5(17.2 \%)$ & $1(3.44 \%)$ & 0.146 \\
\hline $\begin{array}{l}\text { 2-3 salários } \\
\text { mínimo }\end{array}$ & $11(37.93 \%)$ & $3(10.34 \%)$ & $1(3.44 \%)$ & & $13(44.82 \%)$ & $2(6.89 \%)$ & & $12(41.37 \%)$ & $3(10.34 \%)$ & 0 & \\
\hline $\begin{array}{l}\geq 4 \text { salarios } \\
\text { minimo }\end{array}$ & $4(13.79 \%)$ & $1(3.44 \%)$ & 0 & & $5(17.2 \%)$ & 0 & & $4(13.79 \%)$ & $1 \quad(3.44 \%)$ & 0 & \\
\hline
\end{tabular}

${ }^{*}$ Resultados estatisticamente significativos 


\section{ciência
purála}

Tabela 3. Comparação do polimorfismo genético de acordo com características clínicas. Natal RN, 2021.

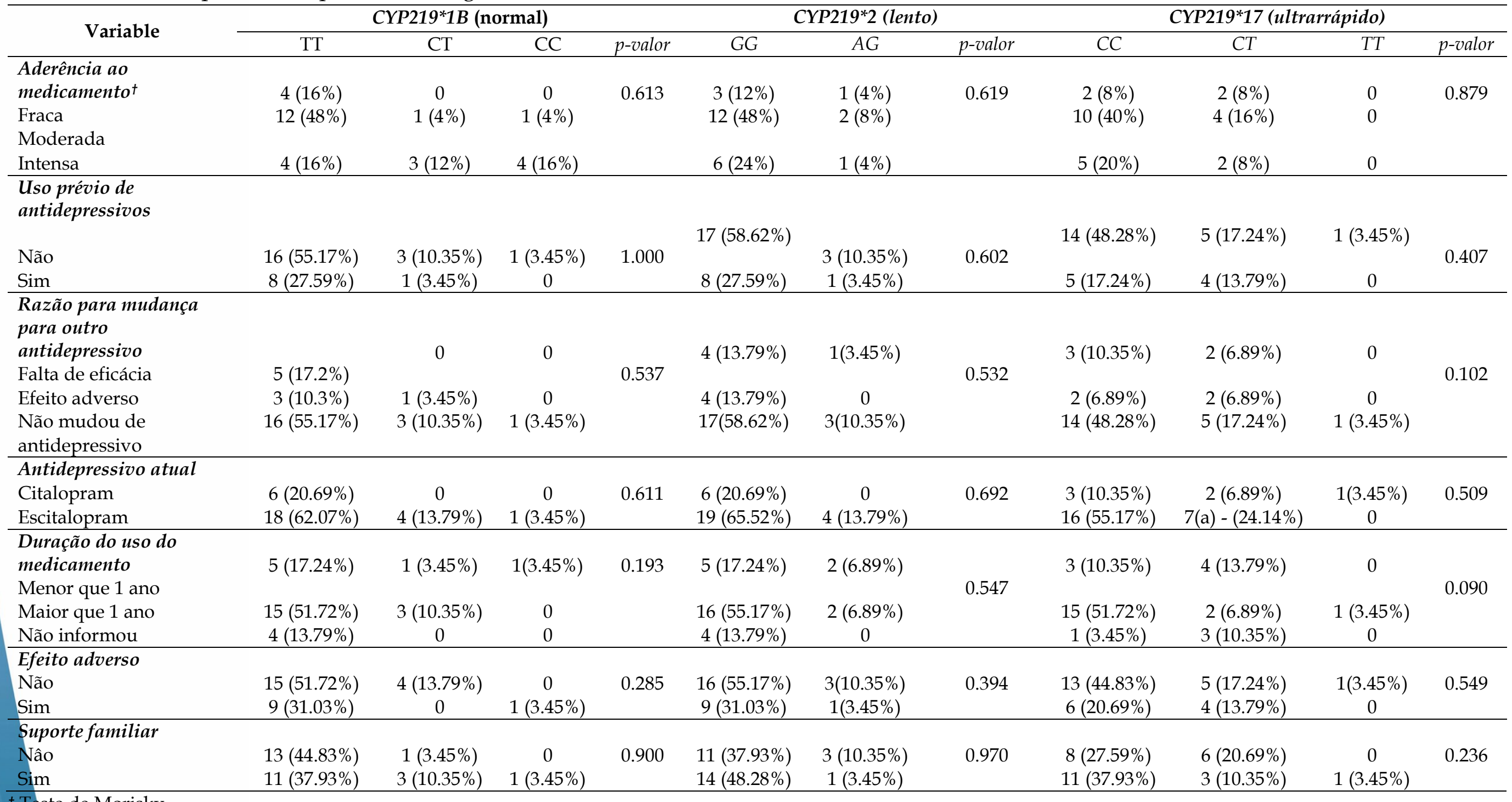


A tabela 3 mostra a comparação genotípica quando as características clínicas. Os dados mostraram não haver associação estatisticamente significativa ( $p>0,05 ;)$. Independentemente do genótipo, a maioria dos indivíduos da presente amostra usava escitalopram e não relatou uso prévio de antidepressivos. Entre os que trocaram de antidepressivos, a maioria dos pacientes relatou que a falta de eficácia foi o principal motivo, independente do polimorfismo genético $(p>0,05)$. Os efeitos adversos foram relatados em 3,45\% dos indivíduos com CYP2C19 * 2 e 13,79\% com CYP2C19 * 17. Os resultados do Teste de Morisky-Green revelaram que a adesão moderada ao tratamento foi predominante para os pacientes CYP2C19 * 2 e CYP219* $17(\mathrm{p}>0,05)$. Uma grande proporção dos indivíduos com polimorfismos CYP2C19 * 2 e CYP2C19 * 17 relatou ausência de apoio familiar $(p>0,05)$.

A Tabela 4 mostra a comparação genotípica quando as escalas de HAM-D e WHOQoL-BREF. Vale ressaltar que entre os 10 pacientes com CYP219 * 17, um apresentou sintomas graves de depressão e dois apresentaram sintomas moderados. Os sintomas fracos de depressão foram predominantes nos pacientes CYP2C19 * 2 e CYP2C19* $17(\mathrm{p}>0,05)$.

Em relação aos resultados do WHOQoL-BREF, uma associação significativa entre o genótipo polimórfico CC de CYP219 * 17 com indivíduos relatando estar satisfeitos com sua saúde, enquanto o genótipo CT foi associado ao status "nem satisfeito/nem insatisfeito" ( $\mathrm{p}=0$,001). A maioria dos indivíduos CYP2C19 * 2 e CYP2C19 * 17 relatou "necessidade de melhorar" ou "regular" em relação aos domínios físico, psicológico, social e ambiental, para a sua qualidade de vida ( $p>0,05)$. 


\section{ciência
pural}

Tabela 4. Comparação do Polimorfismo genético de acordo com a Escala de avaliação de depressão de Hamilton (HAM-D) e avaliação da Qualidade de Vida da Organização Mundial da Saúde - BREF (WHOQoL-BREF). Natal RN, 2021.

\begin{tabular}{|c|c|c|c|c|c|c|c|c|c|c|c|}
\hline \multirow{2}{*}{ Variable } & \multicolumn{4}{|c|}{$C Y P 219 * 1 B$ (normal) } & \multicolumn{3}{|c|}{ CYP219*2 (lenta) } & \multicolumn{4}{|c|}{ CYP219*17 (ultra-rapida) } \\
\hline & $\mathrm{TT}$ & $\mathrm{CT}$ & $\mathrm{CC}$ & p-valor & GG & $A G$ & p-valor & $C C$ & $C T$ & $T T$ & $p$-valor \\
\hline \multicolumn{12}{|l|}{$H A M-D^{\dagger}$} \\
\hline Normal & $9(33.33 \%)$ & $1(3.70 \%)$ & 0 & 0.919 & $9(33.33 \%)$ & $1(3.70 \%)$ & 0.630 & $7(25.92 \%)$ & $2(7.40 \%)$ & $1(3.7 \%)$ & 0.555 \\
\hline Fraco & $8(26.62 \%)$ & $2(7.40 \%)$ & $1(3.75 \%)$ & & $8(29.62 \%)$ & $3(11.11 \%)$ & & $7(25.95 \%)$ & $4(14.81 \%)$ & 0 & \\
\hline Moderado & $2(7.40 \%)$ & 0 & 0 & & $2(7.40 \%)$ & 0 & & 0 & $2(7.40 \%)$ & 0 & \\
\hline Intenso & $2(7.40 \%)$ & $1(3.70 \%)$ & 0 & & $3(11.11 \%)$ & 0 & & $2(7.40 \%)$ & $1(3.7 \%)$ & 0 & \\
\hline Muito intenso & $1(3.70 \%)$ & 0 & 0 & & $1(3.70 \%)$ & 0 & & $1(3.70 \%)$ & 0 & 0 & \\
\hline \multicolumn{12}{|l|}{ WHOQoL-BREF } \\
\hline \multicolumn{12}{|c|}{ Percepção da qualidade } \\
\hline Muito pobre & $2(7 \%)$ & & & 0.692 & $2(7 \%)$ & $1(3.5 \%)$ & 0.692 & & & & 0.355 \\
\hline Pobre & $4(14 \%)$ & $1(3.5 \%)$ & 0 & & $4(14 \%)$ & $1(3.5 \%)$ & & $3(10.7 \%)$ & $2(7 \%)$ & 0 & \\
\hline Nem pobre nem bom & $7(25 \%)$ & $3(11 \%)$ & $1(3.5 \%)$ & & $7(25 \%)$ & $2(7 \%)$ & & $10(35,7 \%)$ & $1(3.5 \%)$ & 0 & \\
\hline Bom & $6(21 \%)$ & 0 & 0 & & $6(21 \%)$ & 0 & & $2(7 \%)$ & $3(10.7 \%)$ & $1(3.5 \%)$ & \\
\hline Muito bom & $4(14 \%)$ & 0 & 0 & & $4(14 \%)$ & & & $2(7 \%)$ & $2(7 \%)$ & 0 & \\
\hline \multicolumn{12}{|l|}{ WHOQoL-BREF } \\
\hline Muito insatisfeito & $1(3.7 \%)$ & 0 & 0 & 0.408 & $1(3.7 \%)$ & 0 & 0.919 & 0 & $1(3.7 \%)$ & 0 & $0.001^{*}$ \\
\hline Insatisfeito & 0 & $1(3.7 \%)$ & 0 & & $1(3.7 \%)$ & 0 & & $1(3.7 \%)$ & 0 & 0 & \\
\hline $\begin{array}{l}\text { Nem satisfeito/nem } \\
\text { insatisfeito }\end{array}$ & $7(26 \%)$ & $1(3.7 \%)$ & $1(3.7 \%)$ & & $8(29.6 \%)$ & $1(3.7 \%)$ & & $4(14.8 \%)$ & $5(18.5 \%)$ & 0 & \\
\hline Satisfeito & $13(48 \%)$ & $2(7.4 \%)$ & 0 & & $12(44.4 \%)$ & $3(11.1 \%)$ & & $12(44.4 \%)$ & $3(11.1 \%)$ & 0 & \\
\hline Muito satisfeito & $1(3.7 \%)$ & 0 & 0 & & $1(3.7 \%)$ & 0 & & 0 & 0 & $1(3.7 \%)$ & \\
\hline \multicolumn{12}{|l|}{ WHOQoL-BREF } \\
\hline \multicolumn{12}{|l|}{ Domínio Físico } \\
\hline Precisa Melhorar & $7(25 \%)$ & $1(35.7 \%)$ & $1(35.7 \%)$ & 0.653 & $7(25 \%)$ & $2(7 \%)$ & 0.678 & $5(17,9 \%)$ & $4(14 \%)$ & 0 & 0.421 \\
\hline Regular & $15(53.6 \%)$ & $3(10.7 \%)$ & 0 & & $16(57 \%)$ & $2(7 \%)$ & & $13(46,4 \%)$ & $4(14 \%)$ & $1(35.7 \%)$ & \\
\hline Boa & $1(3.57 \%)$ & 0 & 0 & & $1(3.57 \%)$ & 0 & & 0 & $1(3.57 \%)$ & 0 & \\
\hline \multicolumn{12}{|l|}{ WHOQoL-BREF } \\
\hline Precisa melhorar & $11(39.3 \%)$ & $2(7 \%)$ & $1(3.57 \%)$ & 0.480 & $12(42.8 \%)$ & $2(7 \%)$ & 0.287 & $10(35.7 \%)$ & $4(14 \%)$ & 0 & 0.515 \\
\hline Regular & $11(39.3 \%)$ & $1(3.57 \%)$ & 0 & & $11(39.3 \%)$ & $1(3.57 \%)$ & & $6(21.4 \%)$ & $5(17.9 \%)$ & $1(35.7 \%)$ & \\
\hline
\end{tabular}




\section{cjênncia
plurâl}

Boa $1(3.57 \%) \quad 1(3.57 \%)$

$1(3.57 \%)$

$1(3.57 \%)$

$2(7 \%)$ 0 0

Dominio Social

Precisa melhorar

$5(17.9 \%) \quad 1(3.57 \%)$

Regular

$12(42.8 \%) \quad 2(7 \%) \quad 1(35.7 \%)$

0.966

$4(14 \%) \quad 2(7 \%)$

$14(50 \%) \quad 1(35.7 \%)$

$4(14 \%) \quad 1(35.7 \%)$

0

$2(7 \%)$

$\begin{array}{ll}0 & 0\end{array}$

$4(14 \%)$

$1(35.7 \%)$

Muito boa

WHOQoL-BREF

Dominio Ambiental

Precisa Melhorar

$8(28.6 \%) \quad 2(7 \%)$

$13(46.4 \%) \quad 1(3.57 \%)$

0
$1(3.57 \%)$

0.627

$2(7 \%)$

0

0.399

$4(14 \%)$

$9(32 \%)$

$2(7 \%)$

$5(17.9 \%)$

0

$(35.7 \%$

$(17.9 \%)$

$2(7 \%)$

0

Regular

$2(7 \%)$

$1(3.57 \%)$

0

$8(28.6 \%)$

$2(7 \%)$

0.393

$7(25 \%)$

9(32\%)

$3(10.7 \%)$

$5(17.9 \%)$

1(3.57\%)

0
$1(3.57 \%)$

0.912

*Resultado estatisticamente significante 


\section{Discussão}

A literatura existente sobre os polimorfismos CYP2C19 em brasileiros com TDM sob tratamento antidepressivo é escassa ${ }^{13}$ e, até onde sabemos, este é o primeiro estudo investigando a relação entre os polimorfismos CYP2C19 * 2 e CYP2C19 * 17 com qualidade de vida e adesão ao tratamento em uma amostra brasileira, específica para o Nordeste, na cidade de Natal.

Outros estudos identificaram a presença desses genótipos e alelos, com maior prevalência da variante CYP2C19*17 do que CYP2C19*213-15, corroborando nossos achados. Vicente et al. ${ }^{16}$ encontraram uma distribuição mais elevada dos alelos CYP2C19*17 na população do Equador quando comparada à população espanhola.

A variação de resposta terapêutica para cada indivíduo com transtorno depressivo maior pode estar relacionada a presença de polimorfismos de nucleotídeo único, principalmente em enzimas metabolizadoras de medicamentos, que podem contribuir para o desenvolvimento de reações adversas ou até mesmo para a ineficácia do tratamento ${ }^{17}$.

Neste contexto, a família das enzimas metabolizadoras têm sido o foco da maioria das pesquisas farmacogenômicas, uma vez que a ocorrência de polimorfismos de nucleotídeo único nas enzimas do citocromo P450 podem comprometer a resposta terapêutica ${ }^{18}$.

Os membros da família da isoenzima CYP2C19 são um dos principais responsáveis pelo metabolismo dos medicamentos utilizados no tratamento de pacientes com transtorno depressivo maior, dentre eles o citalopram e escitalopram (3). Polimorfismos do gene CYP2C19, que codifica essa família de isoenzima, têm sido relacionados com alterações na metabolização desses medicamentos, comprometendo assim a sua eficácia ${ }^{19}$.

Dados socioeconômicos e demográficos revelaram que os polimorfismos CYP219*2 e CYP2C19*17 foram prevalentes em mulheres, com idade entre 18-44 anos, m 1-11 anos de estudo e baixa renda, ou em situação de desemprego. Esse perfil flete as dificuldades da população feminina de baixa renda no Brasil, imersa em um ário de falta de igualdade de oportunidades para mulheres e homens, violência nciosa contra a mulher e dificuldade de acesso aos serviços de saúde 20-22. 


\section{ciência
piural}

A distribuição dominante do CYP2C19*17 em comparação com o CYP2C19*2 pode indicar um perfil de aumento da atividade enzimática, que resulta em um intervalo posológico, para os antidepressivos em estudo, descoberto, com presença de sintomas depressivos, conforme demonstrado pelos escores moderados e intensos relatados em HAM-D nos pacientes portadores do CYP2C19*17. O polimorfismo CYP2C19*17 é responsável pela atividade ultrarrápida das enzimas que metabolizam o citalopram e o escitalopram. Foi comprovado que indivíduos que expressam esta variante do gene apresentam menor concentração plasmática do princípio ativo quando comparados àqueles que não possuem esse polimorfismo, o que pode resultar em concentrações subterapêuticas, condição que pode comprometer o tratamento farmacológico justamente por não ser eficaz na redução dos sintomas da doença ${ }^{15}$.

Aldrich et al. ${ }^{23}$ demonstraram uma disparidade nos resultados para jovens com transtornos depressivos e/ou ansiedade tratados com doses padronizadas de es/citalopram, sem considerar o status do metabolizador CYP2C19. Brito \& Ghedini13 descobriram que os pacientes brasileiros com TDM com a variante CYP2C19*17 (ultrarrápida) tinham doses de escitalopram significativamente maiores do que os metabolizadores extensos e intermediários e sugeriram que o alelo ultrarrápido CYP2C19*17 parece exercer influência na baixa resposta ao escitalopram, mesmo em doses mais altas.

Em relação à adesão ao tratamento, a maioria dos pacientes CYP2C19*17 (50\%) teve adesão moderada, $25 \%$ teve baixa adesão e apenas $25 \%$ teve boa adesão. Esta adesão insatisfatória adicionada à probabilidade de falha terapêutica devido ao rápido metabolismo de Es/citalopram, espera-se uma eficácia do tratamento muito baixa como resultado. Aldrich et al. ${ }^{23}$ sugeriram que os metabolizadores CYP2C19 apresentam tolerabilidade reduzida a es/citalopram, o que pode resultar em risco aumentado de descontinuação, em comparação com metabolizadores ultrarrápidos. De acordo com Wessels et al. ${ }^{24}$, a má adesão ao escitalopram por pacientes com depressão geralmente ocorre devido à descontinuação precoce do tratamento e xecução diária subótima do regime prescrito.

Estima-se que a não adesão ao tratamento da depressão ocorra em 40 a $75 \%$ dos cientes $^{25}$. Nesse sentido, o paciente depressivo parece necessitar de uma postura 
deliberada de apoio familiar e social para lembrá-lo de sua importância pessoal e das possibilidades de boas perspectivas de vida, o que proporcionaria um estímulo externo para a continuidade do tratamento. A falta de disposição, pensamentos pessimistas, diminuição da energia e fadiga, muitas vezes presentes como sintomas depressivos, podem encontrar endosso na postura resistente ou não colaborativa dos familiares.

A relação entre os polimorfismos genéticos e o instrumento WHOQoL-BREF listou duas variáveis principais: (1) como o indivíduo avalia sua qualidade de vida, e (2) com que frequência o indivíduo tem sentimentos negativos, como mau humor, desespero, ansiedade e depressão. Nossos resultados indicaram que a maioria dos metabolizadores ultrarrápidos relatou não estar nem satisfeita / nem insatisfeita com a satisfação com a saúde. Além disso, os pacientes CYP219*2 e CYP219*17 também apresentam pontuações baixas nos domínios físico, psicológico, social e ambiental do WHOQoL-BREF. Espera-se que, nesses pacientes, o fármaco seja metabolizado mais rapidamente, resultando em janelas de subdosagem, o que pode influenciar nas recaídas e na ineficácia do tratamento, além de reduzir a qualidade de vida .

Embora seja um estudo pioneiro no tema, faz-se necessário esclarecer que o número de amostra desenhado para o estudo, 50 pacientes, não foi alcançado na coleta, devido aos critérios de exclusão e por alguns não aceitarem participar do estudo. Durante a comparação dos perfis genotípicos não foram encontradas diferenças estatísticas em relação a algumas variáveis, o que pode estar relacionado ao tamanho da amostra.

$\mathrm{Na}$ prática clínica, o principal objetivo da genotipagem é personalizar o tratamento para cada tipo de paciente de acordo com suas características, fornecendo o medicamento mais adequado e a dose mais tolerada. Portanto, investigações ou estratégias mais diversificadas, incluindo o estudo envolvendo variantes de baixa frequência, são de grande utilidade para desenvolver uma padronização para futuras plicações de testes clínicos ${ }^{8}$.

\section{nclusões}


Os resultados fornecem dados preliminares mostrando uma maior prevalência de CYP219*17 na amostra, com adesão moderada ao tratamento. Mesmo sob efeito da medicação, os indivíduos apresentavam sintomas moderados a intensos de depressão e relataram uma indefinição na satisfação da sua qualidade de vida, considerando os domínios físico, psicológico, social e ambiental como regular ou precisa melhorar. Assim concluímos que apesar da complexidade do desenvolvimento de pesquisas na área de farmacogenética, os estudos de genotipagem oferecem uma valiosa ferramenta de apoio ao tratamento tradicional do TDM.

\section{Agradecimentos}

À Profa Dra Adriana Augusto Rezende, Laboratório de Biologia Molecular LABIOMOL, PPgCF/UFRN. Aos Médicos residentes e profissionais de Saúde do Setor de Psiquiatria e Psicodermatologia do Hospital Universitário Onofre Lopes/HUOLUFRN. Aos profissionais do Laboratório de Análises clínicas do Hospital Universitário Onofre Lopes/HUOL-UFRN. À Pro-reitoria de pós-graduação da UFRN. Ao professor Dr José Perez Urizar , Universidad Autonoma de San Luiz de Potosi, México.

\section{Referências}

1 Malhi GS, Mann JJ. Depression. Lancet (London, England). 2018;392(10161):2299-312.

2 Munhoz TN, Nunes BP, Wehrmeister FC, Santos IS, Matijasevich A. A nationwide population-based study of depression in Brazil. J Affect Disord. 2016;192:226-33.

Carvalho AF, Sharma MS, Brunoni AR, Vieta E, Fava GA. The Safety, Tolerability and Risks Associated with the Use of Newer Generation Antidepressant Drugs: A Critical Review of the Literature. Psychother Psychosom. 2016;85(5):270-88.

Al-Harbi KS. Treatment-resistant depression: therapeutic trends, challenges, future directions. Patient Prefer Adherence. 2012;6:369-88. 
5 Kendrick T, Taylor D, Johnson CF. Which first-line antidepressant? British Journal of General Practice. 2019;69(680):114.

6 Tsai MH, Lin KM, Hsiao MC, Shen WW, Lu ML, Tang HS, et al. Genetic polymorphisms of cytochrome $\mathrm{P} 450$ enzymes influence metabolism of the antidepressant escitalopram and treatment response. Pharmacogenomics. 2010;11(4):537-46.

7 Lee SJ. Clinical Application of CYP2C19 Pharmacogenetics Toward More Personalized Medicine. Front Genet. 2012;3:318.

8 Li-Wan-Po A, Girard T, Farndon P, Cooley C, Lithgow J. Pharmacogenetics of CYP2C19: functional and clinical implications of a new variant CYP2C19*17. Br J Clin Pharmacol. 2010;69(3):222-30.

9 Rudberg I, Mohebi B, Hermann M, Refsum H, Molden E. Impact of the ultrarapid CYP2C19*17 allele on serum concentration of escitalopram in psychiatric patients. Clin Pharmacol Ther. 2008;83(2):322-7.

10 Hamilton M. A rating scale for depression. J Neurol Neurosurg Psychiatry. 1960;23(1):56-62.

11 Morisky DE, Green LW, Levine DM. Concurrent and predictive validity of a self-reported measure of medication adherence. Med Care. 1986;24(1):67-74.

12 Fleck MP, Louzada S, Xavier M, Chachamovich E, Vieira G, Santos L, et al. Aplicação da versão em português do instrumento abreviado de avaliação da qualidade de vida "WHOQOL-bref". Rev Saúde Públ. 2000;34(2):178-83.

13 Brito RB, Ghedini PC. CYP2C19 polymorphisms and outcomes of Escitalopram treatment in Brazilians with major depression. Heliyon. 2020;6(5):e04015.

14 Uckun Z, Baskak B, Ozel-Kizil ET, Ozdemir H, Devrimci Ozguven H, Suzen HS. The impact of CYP2C19 polymorphisms on citalopram metabolism in patients with major depressive disorder. J Clin Pharm Ther. 2015;40(6):672-9.

15 Hicks JK, Bishop JR, Sangkuhl K, Müller DJ, Ji Y, Leckband SG, et al. Clinical Pharmacogenetics Implementation Consortium (CPIC) Guideline for CYP2D6 and CYP2C19 Genotypes and Dosing of Selective Serotonin Reuptake Inhibitors. Clin Pharmacol Ther. 2015;98(2):127-34.

Vicente J, Gonzalez-Andrade F, Soriano A, Fanlo A, Martinez-Jarreta B, Sinues Genetic polymorphisms of CYP2C8, CYP2C9 and CYP2C19 in Ecuadorian Mestizo d Spaniard populations: a comparative study. Mol Biol Rep. 2014;41(3):1267-72. 
17 De Vos A, Van Der Weide J, Loovers HM. Association between CYP2C1917 and metabolism of amitriptyline, citalopram and clomipramine in Dutch hospitalized patients. Pharmacogenomics J. 2011;11(5):359-67.

18 Lynch T, Price A. The Effect of Cytochrome P450 Metabolism on Drug Response, Interactions, and Adverse Effects. Am Fam Physician. 2007;76(3):391-6.

19 Ng I, Greenblatt HK, Greenblatt DJ. Stereo-Psychopharmacology: The Case of Citalopram and Escitalopram. Clin Pharmacol Drug Dev. 2016;5(5):331-5.

20 Madalozzo R. Occupational segregation and the gender wage gap in Brazil: an empirical analysis. Economia Aplicada. 2010;14(2):147-68.

21 Leite MTS, Figueiredo MFS, Dias OV, Vieira MA, Souza e Souza LP, Mendes DC. Reports of violence against women in different life cycles. Revista LatinoAmericana de Enfermagem. 2014;22(1):85-92.

22 Massuda A, Hone T, Leles FAG, de Castro MC, Atun R. The Brazilian health system at crossroads: progress, crisis and resilience. BMJ Glob Health. 2018;3(4):e000829-e.

23 Aldrich SL, Poweleit EA, Prows CA, Martin LJ, Strawn JR, Ramsey LB. Influence of CYP2C19 Metabolizer Status on Escitalopram/Citalopram Tolerability and Response in Youth With Anxiety and Depressive Disorders. Front Pharmacol. 2019;10:99.

24 Wessels A, Jin Y, Pollock B, Frank E, Lange A, Vrijens B, et al. Adherence to escitalopram treatment in depression: a study of electronically compiled dosing histories in the 'Depression: the search for phenotypes' study. Int Clin Psychopharmacol. 2012;27(6):291-297

25 Pampallona S, Bollini P, Tibaldi G, Kupelnick B, Munizza C. Patient adherence in the treatment of depression. Br J Psychiatry. 2002;180:104-9. 